\title{
Hedgehogs of constant width and equichordal points
}

\author{
by Yves Martinez-Maure (Rueil-Malmaison)
}

\begin{abstract}
We give a characterization of convex hypersurfaces with an equichordal point in terms of hedgehogs of constant width.
\end{abstract}

I. Introduction and statement of results. Let $K$ be a convex body in $(n+1)$-dimensional Euclidean space $\mathbb{E}^{n+1}$ and let $S$ be its boundary. An interior point $o$ of $K$ is called an equichordal point of $S$ if all chords of $S$ passing through $o$ have the same length.

A famous unsolved problem is whether there exist plane convex curves with two equichordal points. A discussion of this problem, first raised by Fujiwara [2] and independently by Blaschke, Rothe and Weitzenböck [1], is given by Klee ([4] and [5]). Wirsing [10] proved (assuming their existence) that such curves are analytic. Petty and Crotty [8] have proved the existence of Minkowski spaces of arbitrary dimension in which there are convex hypersurfaces with exactly two equichordal points.

Let $S$ be a smooth convex hypersurface in $\mathbb{E}^{n+1}$. The pedal hypersurface $P(S)$ with respect to an interior point $o$ is defined as follows: for each $m \in S$ the point $P(m)$ is the foot of the perpendicular from the point $o$ to the tangent hyperplane of $S$ at $m$. If $S$ is of constant width then $P(S)$ has $o$ as an equichordal point, but $P(S)$ is not necessarily convex.

Conversely, Kelly [3] has shown that if a plane convex curve $C$ has $O$ as an equichordal point, then $C$ is the pedal curve with respect to $o$ of a curve $P^{-1}(C)$ with a kind of constant width. This curve $P^{-1}(C)$, called the negative pedal of the curve $C$, is not necessarily convex.

In this paper, we prove the following generalization to hypersurfaces.

THEOREM 1. If $S$ is a smooth convex hypersurface with an equichordal point o, then $S$ is the pedal hypersurface with respect to o of a hypersurface

1991 Mathematics Subject Classification: Primary 52A20.

Key words and phrases: convex body, hypersurface, pedal, equichordal, hedgehog. 
$P^{-1}(S)$ with a kind of constant width. More precisely, $P^{-1}(S)$ is a hedgehog of constant width as defined below.

Definition 1. For any $f \in C^{\infty}\left(\mathbb{S}^{n} ; \mathbb{R}\right)$, we define the hedgehog with supporting function $f$ as the envelope $\mathcal{H}_{f}$ of the family of hyperplanes defined by the equations

$$
\langle x, p\rangle=f(p)
$$

(these hyperplanes are called the supporting hyperplanes of $\mathcal{H}_{f}$ ). In other words, $\mathcal{H}_{f}$ is the hypersurface (with possible singularities) parametrized by

$$
x_{f}: \mathbb{S}^{n} \rightarrow \mathcal{H}_{f}, \quad p \mapsto x_{f}(p),
$$

where $x_{f}(p)=f(p) p+(\operatorname{grad} f)(p)$ is the unique solution of the system

$$
\langle x, p\rangle=f(p), \quad\langle x, \cdot\rangle=d f_{p}(\cdot) .
$$

R e m a r k. When $\mathcal{H}_{f}$ has a well defined tangent hyperplane at $x_{f}(p)$, say $T$, then $T$ is defined by the equation $\langle x, p\rangle=f(p)$ : the unit vector $p$ is normal to $T$ and $f(p)$ may be interpreted as the signed distance from the origin to $T$. Thus, any smooth part of $\mathcal{H}_{f}$ inherits a natural transverse orientation for which $x_{f}$ is the reverse Gauss map. A singularity-free hedgehog is simply a convex hypersurface. For a general study of hedgehogs see R. Langevin, G. Levitt and H. Rosenberg [6].

DEFINITION 2. The hedgehog with supporting function $f$ is said to be of constant width if the distance between two parallel supporting hyperplanes is constant, that is, if $f(p)+f(-p)$ is constant on $\mathbb{S}^{n}$.

We next prove the following results.

THEOREM 2. Let $S$ be a smooth convex hypersurface with the origin as an equichordal point. The negative pedal hypersurface of $S$ with respect to the origin is convex if and only if the hypersurface obtained from $S$ by inversion with respect to $\mathbb{S}^{n}$ is convex.

THEOREM 3. Let $\mathcal{H}_{f}$ be a hedgehog of constant width such that $f$ is never zero. Then the pedal hypersurface of $\mathcal{H}_{f}$ with respect to the origin is a smooth hypersurface with the origin as an equichordal point. Furthermore, $P\left(\mathcal{H}_{f}\right)$ is convex if and only if $1 / f$ is the supporting function of a convex hedgehog.

Note that these problems are related to equireciprocal points of convex bodies (see [4]).

\section{Proof of results}

Proof of Theorem 1. Assume without loss of generality that $o$ is the origin. Since $S$ is starlike relative to $o, S$ has a parametrization of the form

$$
X: \mathbb{S}^{n} \rightarrow S, \quad p \mapsto g(p) p,
$$


where the function $g$ is $>0$. The condition that $S$ have the origin as an equichordal point is simply that $g$ be of the form $g=h+r$, where $r$ is a constant and $h$ is a function such that

$$
\forall p \in \mathbb{S}^{n}, \quad h(-p)=-h(p) .
$$

Note that the hedgehog $\mathcal{H}_{h}$ can be considered as a hedgehog of zero width: such a hedgehog is said to be projective. For a study of projective hedgehogs, see $[7]$.

Since $(\operatorname{grad} g)(p) \in T_{p} \mathbb{S}^{n}$, it follows from the parametrization $x_{g}(p)=$ $g(p) p+(\operatorname{grad} g)(p)$ of the hedgehog $\mathcal{H}_{g}$ that $S$ is the pedal hypersurface of $\mathcal{H}_{g}$ with respect to $o$. Furthermore, this hedgehog $\mathcal{H}_{g}$ is of constant width since the distance $d(p)$ between the two supporting hyperplanes of $\mathcal{H}_{g}$ which are orthogonal to $p \in \mathbb{S}^{n}$ is given by

$$
d(p)=g(p)+g(-p)=2 r=\text { const. }
$$

Theorems 2 and 3 are based on the following result (see for example the book by R. Schneider [9], Sections 1.6 and 1.7).

LEMMA. Let $h_{L}\left(\right.$ resp. $\left.\varrho_{L}\right)$ denote the supporting (resp. radial) function of a convex body $L$ with the origin as an interior point. If a convex body $K$ has the origin as an interior point, then its polar body $K^{*}$ also has the origin as an interior point, and we have

$$
h_{K^{*}}=1 / \varrho_{K} \quad \text { and } \varrho_{K^{*}}=1 / h_{K} .
$$

Proof of Theorem 2. Let $\Sigma$ and $\Sigma^{*}$ denote respectively the negative pedal hypersurface $P^{-1}(S)$ and the hypersurface obtained from $S$ by inversion with respect to $\mathbb{S}^{n}$. We can deduce from the Lemma that if $\Sigma$ or $\Sigma^{*}$ is convex, then $\Sigma$ and $\Sigma^{*}$ are the boundaries of polar bodies $K$ and $K^{*}$. Theorem 2 follows immediately.

Proof of Theorem 3. The pedal hypersurface of $\mathcal{H}_{f}$ is the smooth hypersurface parametrized by

$$
X: \mathbb{S}^{n} \rightarrow S, \quad p \mapsto f(p) p
$$

which has the origin as an equichordal point since $f(p)+f(-p)$ is constant. Furthermore, we can deduce from the Lemma that if $P\left(\mathcal{H}_{f}\right)$ or $\mathcal{H}_{1 / f}$ is convex, then $P\left(\mathcal{H}_{f}\right)$ and $\mathcal{H}_{1 / f}$ are the boundaries of polar bodies. Theorem 3 follows immediately.

\section{References}

[1] W. Blaschke, H. Rothe und R. Weitzenböck, Aufgabe 552, Arch. Math. Phys. 27 (1917), 82.

[2] M. Fujiwara, Über die Mittelkurve zweier geschlossenen konvexen Kurven in Bezug auf einen Punkt, Tôhoku Math. J. 10 (1916), 99-103. 
[3] P. J. Kelly, Curves with a kind of constant width, Amer. Math. Monthly 64 (1957), 333-336.

[4] V. Klee, Can a plane convex body have two equichordal points?, Amer. Math. Monthly 76 (1969), 54-55.

[5] - Some unsolved problems in plane geometry, Math. Mag. 52 (3) (1979), 131-145.

[6] R. Langevin, G. Levitt et H. Rosenberg, Hérissons et multihérissons (Enveloppes paramétrées par leur application de Gauss), in: Singularities (Warsaw, 1985), Banach Center Publ. 20, PWN, Warszawa, 1988, 245-253.

[7] Y. Martinez-Maure, Sur les hérissons projectifs (enveloppes paramétrées par leur application de Gauss), Bull. Sci. Math., to appear.

[8] C. M. Petty and J. M. Crotty, Characterization of spherical neighborhoods, Canad. J. Math. 22 (1970), 431-435.

[9] R. Schneider, Convex Bodies: The Brunn-Minkowski Theory, Cambridge Univ. Press, 1993.

[10] E. Wirsing, Zur Analytizität von Doppelspeichkurven, Arch. Math. (Basel) 9 (1958), 300-307.

1 , rue Auguste Perret

92500 Rueil-Malmaison, France 